\title{
Stability of Grid Amplifiers
}

\author{
Cheh-Ming Liu, Michael P. De Lisio, Member, IEEE, Alina Moussessian, \\ and David B. Rutledge, Fellow, IEEE
}

\begin{abstract}
We present a stability model for quasi-optical grid amplifiers. This model is useful for predicting and suppressing the common-mode oscillations that often occur in amplifier grids. Three stabilization techniques will be discussed. The first technique uses a capacitor to stabilize the grid. The second approach employs resistance to suppress the oscillations. The final technique stabilizes the grid by reducing the on-chip commonmode resistance, allowing greatly increased amplifier efficiencies. Experimental evidence will be presented to confirm the validity of our stability model.
\end{abstract}

Index Terms - Grid amplifiers, quasi-optics, stability.

\section{INTRODUCTION}

A GRID amplifier, shown in Fig. 1, is an array of closely spaced differential transistor pairs. A horizontally polarized input beam excites RF currents on the input leads of the grid. This drives the transistor pair in the differential mode. Currents on the output leads radiate a vertically polarized output beam. The cross-polarized input and output prevents spurious feedback oscillations. Metal-strip polarizers confine the beam to the forward direction and independently tune the grid's input and output. Hybrid microwave grid amplifiers have been demonstrated using MESFET's [1], heterojunction bipolar transistors (HBT's) [2], [3], and pseudomorphic high electron-mobility transistors (pHEMT's) [4], [5]. Millimeterwave HBT [6] and pHEMT [7] grids are among the few successful monolithic quasi-optical amplifiers.

Stability is a vital concern for any amplifier. This may be especially true for quasi-optical amplifiers, where many oscillation modes may exist due to the large number of devices involved. In a grid amplifier, the problem is compounded by the rather close device spacing. Typical spacings range from $\lambda_{0} / 10$ to $\lambda_{0} / 3$, where $\lambda_{0}$ is the free-space wavelength. Individual elements tend to be strongly coupled. The dielectric substrate is another mechanism that will contribute to the interelement coupling. Griffin [8] pointed out that substrate-mode excitation is responsible for the poor radiation patterns of grid oscillators. In grid oscillators, substrate-mode excitation can be minimized through careful choice of the substrate thickness and element spacing [9]. Many grid amplifiers have, in fact, suffered from oscillations of some kind [1]-[5]. Often, these

Manuscript received November 11, 1997; revised March 4, 1998. This work was supported by the U.S. Air Force Material Command/Rome Laboratory and by the U.S. Army Research Offcie under a MURI Grant to Caltech.

C.-M. Liu is with the Rockwell Science Center, Rockwell International Corporation, Thousand Oaks, CA 91385 USA.

M. P. De Lisio is with the Department of Electrical Engineering, University of Hawaii at Manoa, Honolulu, HI 96822 USA.

A. Moussessian is with the Radar Science and Engineering Section, Jet Propulsion Laboratory, Pasadena, CA 91109-8099 USA.

D. B. Rutledge is with the Department of Electrical Engineering, California Institute of Technology, Pasadena, CA 91125 USA.

Publisher Item Identifier S 0018-9480(98)04039-3.

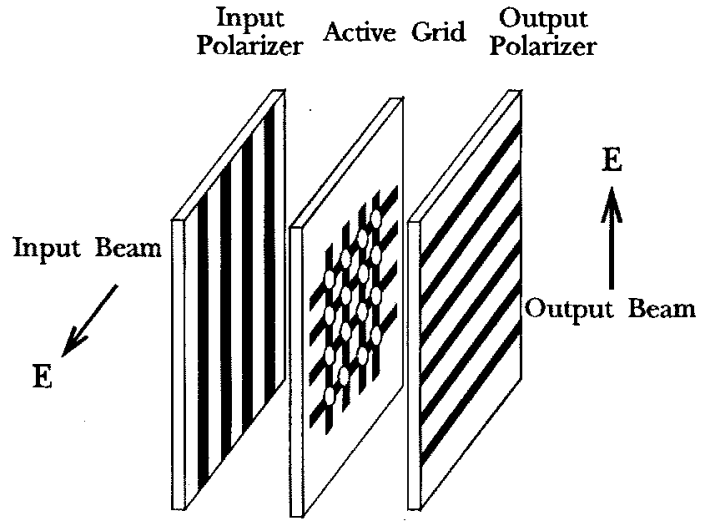

Fig. 1. A grid amplifier.

oscillations appear at moderate bias levels. If these instabilities cannot be suppressed, the grid will be limited to low bias currents, severely restricting the gain and output power. A reliable stability model is of paramount importance.

\section{INSTABILITY CHARACTERIZATION}

Most of our stability modeling is the result of the systematic study of a hybrid HBT grid amplifier [3]. The grid was a $4 \times 4$ array of HBT differential pairs mounted on a 50-mil Duroid substrate with a relative dielectric constant of 10.8. Fig. 2(a) shows the unit cell. Collector and emitter bias was provided by the thin "hairpin" bias lines. These lines were designed to have a parallel resonance near the $10-\mathrm{GHz}$ operating frequency. Etched capacitive gaps in the base leads were used to match the grid's input. Fig. 2(b) shows a schematic of the active chip. Each AlGaAs/GaAs HBT had an effective area of $40 \mu \mathrm{m}^{2}$. The $2-\mathrm{k} \Omega$ feedback resistor self-biases the base from the collector. The two 750- $\Omega$ emitter resistors were intended to reduce the pair's common-mode gain. The HBT chips were fabricated by Rockwell International, Thousand Oaks, CA.

This grid was plagued by instability. A 7.8-GHz spurious oscillation appeared when the collector bias current exceeded $2.4 \mathrm{~mA}$ per transistor. This is much below the HBT's optimum bias current of $20 \mathrm{~mA}$. At the onset of oscillation, the amplifier's peak gain was less than $4 \mathrm{~dB}$.

Investigations of this instability revealed several interesting properties. The oscillation was quite insensitive to changes in the elements external to the active surface. For example, the positions of the external polarizers, tuning slabs, or the thickness of the substrate had little effect on the oscillation frequency or power. On the other hand, the oscillation was very sensitive to changes made in the unit cell itself. For example, shorting the input matching gap capacitor with bond wires caused the oscillation frequency to shift to $4.8 \mathrm{GHz}$. 


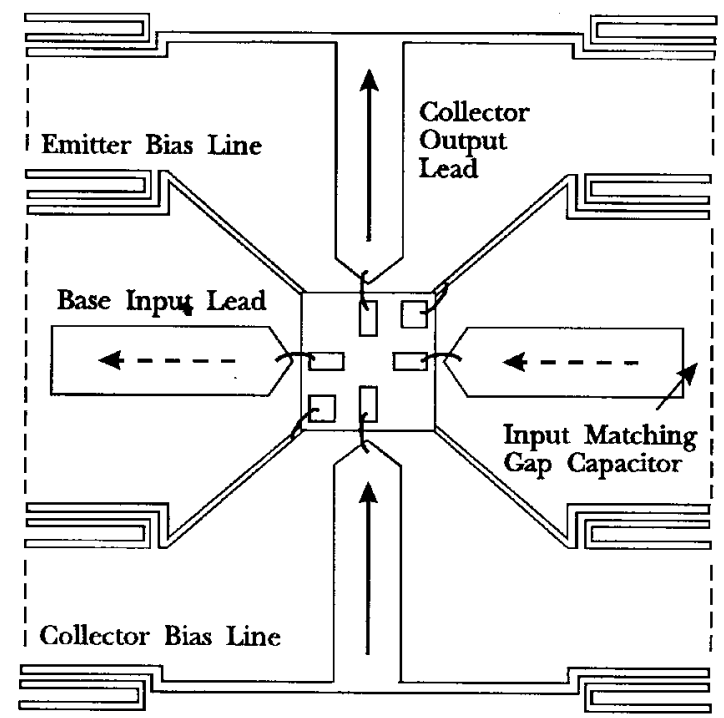

(a)

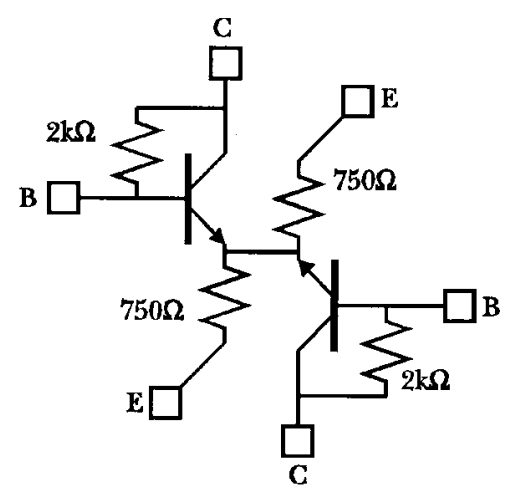

(b)

Fig. 2. (a) Unit cell of the 16-element hybrid HBT grid. The cell is $8 \mathrm{~mm}$ on a side. (b) Schematic of the HBT differential pair.

These phenomena indicate that the oscillation mechanism primarily depends on the array's transverse properties, as opposed to the amplifier's longitudinal properties.

Further clues about the nature of the oscillation can be inferred from measurements of the grid's radiation pattern. Fig. 3 plots the radiation pattern in the amplifier's output $H$-plane. Peaks in the radiation pattern occurred in the plane of the amplifier. The power density radiated normally to the array was $6 \mathrm{~dB}$ less. The radiated power in the plane of the grid was concentrated on the side of the emitter bias supply. Furthermore, the radiated power was strongly polarized along the direction of the grid's output collector leads.

This evidence led to the conclusion that the oscillation was a common-mode one with dominant collector currents. The current distribution is proposed in Fig. 4(a). Adjacent cells are $180^{\circ}$ out of phase, giving the array a "checkerboard" distribution. This current distribution will not radiate well normal to the grid, but may couple well to substrate modes and radiate into free space from the edges of the array. Further support for this conclusion lies in the fact that the 8-mm unit cell was exactly one-half of a guided wavelength at $7.8 \mathrm{GHz}$, assuming an effective relative dielectric constant of 5.8. Once the oscillation has been characterized, a stability model can be produced.

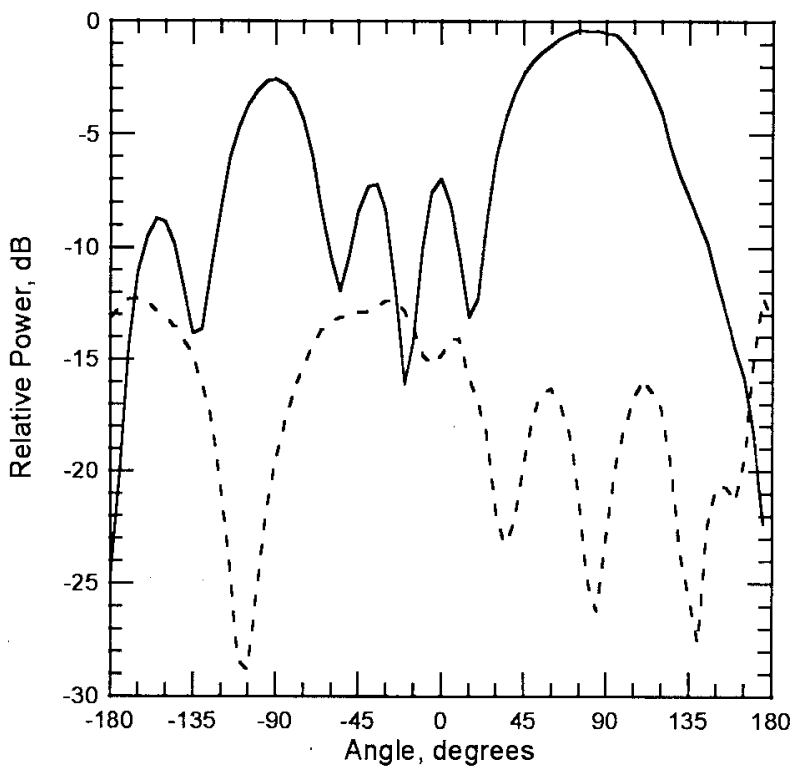

Fig. 3. Measured radiation pattern of the spurious oscillation. The pattern is taken in the amplifier's output $H$-plane. The solid line is polarized along the grid's output collector leads. The dashed line is polarized along the input base leads.

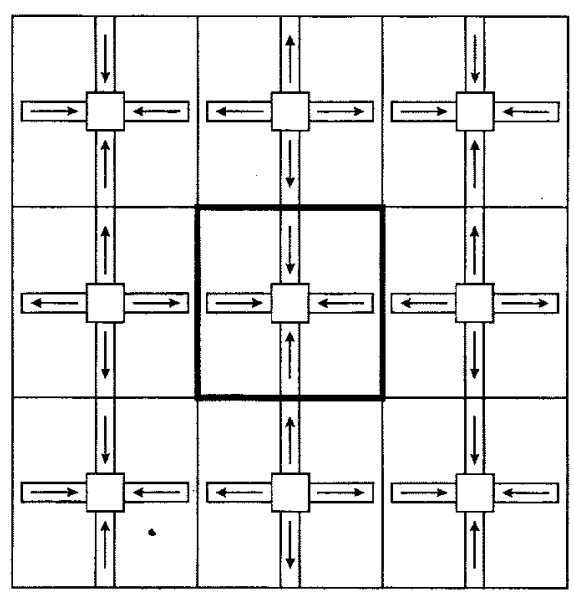

(a)

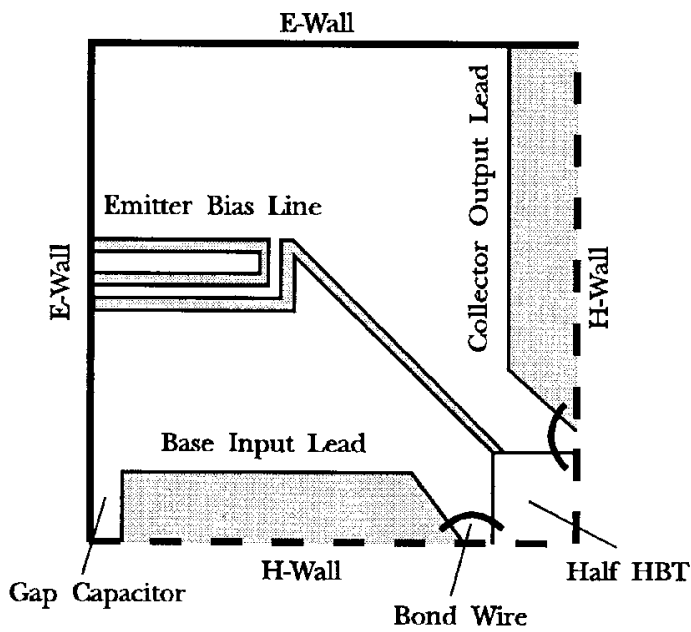

(b)

Fig. 4. (a) Deduced current distribution on the array. A unit cell is highlighted in the center. (b) Quarter-unit cell used for stability modeling. The cell has two electric walls and two magnetic walls. 


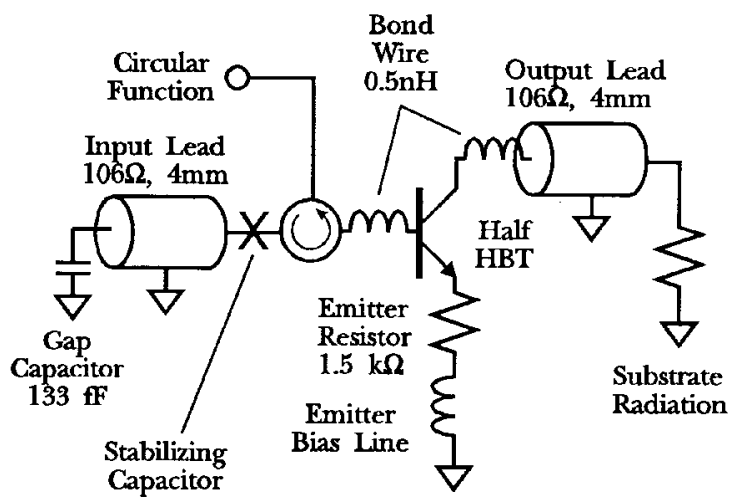

Fig. 5. Transmission-line equivalent circuit for the hybrid HBT grid oscillation.

\section{STABILITY MODELING}

The first step in modeling the checkerboard common-mode oscillation of Fig. 4(a) is to recognize that the symmetries of the current distribution can be exploited to define a unit cell. This unit cell will be bounded on all four sides by electric walls, where the tangential electric field must vanish. The full unit cell can then be divided into quarters, with each quarter cell consisting of two electric walls and two magnetic walls. A quarter cell is illustrated in Fig. 4(b). Because each unit cell has two transistors, the quarter cell will contain one-half of a transistor. The $Y$-parameters of the half transistor are onehalf of those for a single HBT; two half transistors wired in parallel constitute a full device.

Fig. 5 shows a transmission-line equivalent circuit for the oscillation. Because the oscillation occurs in the transverse dimension, the input and output leads are modeled as transmission lines. The electrical length of these lines is calculated using the physical size of the unit cell and the mean dielectric constant for air and the Duroid substrate. The impedance of the transmission lines is chosen empirically to fit the observed oscillation frequency. Inductive bond wires connect the device to the leads. Coupling to the substrate modes is modeled as a resistance in the output lead. Our experience has shown that this substrate-mode radiation resistance is quite low, and larger resistance values tend to reduce the potential for oscillation. This resistor may be replaced with a short for a worst-case analysis. In addition, the on-chip common-mode emitter resistors must be included. The quarter-cell emitter resistance is $1.5 \mathrm{k} \Omega$. The emitter bias line is modeled as an inductor. For the specific case of the hybrid HBT oscillator, the hairpin emitter bias line reactance at the oscillation frequency is negligible compared to the emitter resistance.

The circuit in Fig. 5 may be analyzed using a variety of techniques. One approach that is especially intuitive and useful is the technique suggested by Martinez and Compton [10]. An ideal circulator is inserted into the circuit. The reflection from the circulator is defined as the circular function. The circular function can be thought of as a measure of the open-loop gain of the circuit. Stability theory dictates that the circuit will oscillate if the circular function is real and greater than unity. The simulated circular function is plotted in Fig. 6. An oscillation is predicted at the observed $7.8 \mathrm{GHz}$. The zerophase loop gain is $5.4 \mathrm{~dB}$. In agreement with observation,

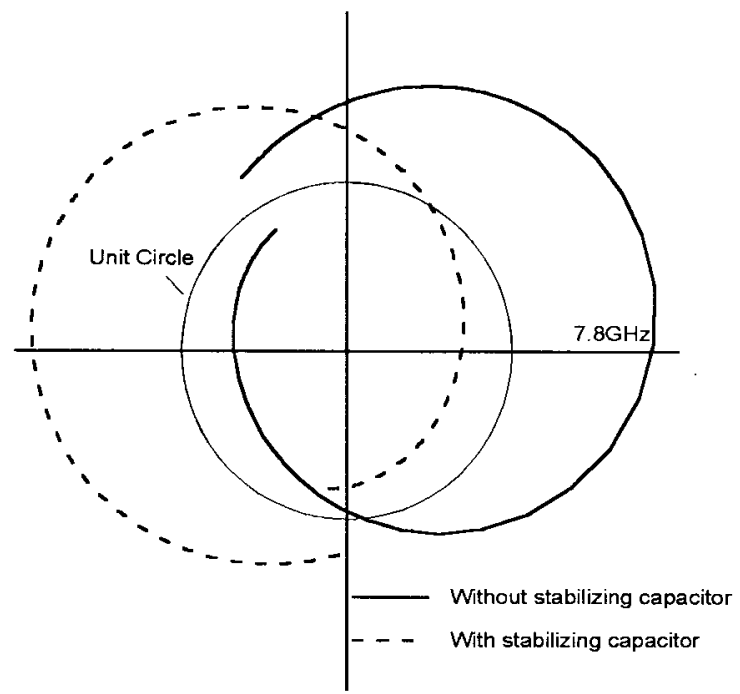

Fig. 6. Simulated circular function of the hybrid HBT grid amplifier with and without stabilizing capacitors.

shorting the matching gap capacitor will lower the oscillation frequency. This instability must be suppressed for the amplifier to perform properly.

\section{CApacitive Stabilization}

To stabilize the grid, series chip capacitors were added in the base input lead, directly adjacent to the active chip. One-half of this stabilizing capacitance will appear at the node marked with an "X" in Fig. 5. This extra capacitive reactance will provide additional phase shift, rotating the circular function in the complex plane. Thereby, the zero-phase magnitude is reduced. The circular function with $0.1-\mathrm{pF}$ stabilizing capacitors is also plotted in Fig. 6. The model predicts the circuit is now stable with a gain margin of $3.4 \mathrm{~dB}$ and a phase margin of $70^{\circ}$. Experimentally, the grid with the $0.1-\mathrm{pF}$ stabilizing capacitors could be biased as high as $10 \mathrm{~mA}$ per transistor without oscillating. The peak gain of the stabilized amplifier was 8.5 $\mathrm{dB}$, and the higher bias current will allow greater output power. To further validate the stability model, the amplifier's stability was examined as a function of stabilizing capacitance. Table I summarizes the results. In general, theory and experiment indicate that lower capacitance results in a more stable amplifier.

This stability model was used as a design tool to stabilize a monolithic millimeter-wave HBT grid amplifier [6]. This array integrated $36 \mathrm{HBT}$ pairs, and was fabricated by Rockwell International. Without stabilizing base capacitors, theory predicts that this array would oscillate at $21.3 \mathrm{GHz}$, as shown in Fig. 7. The zero-phase circular function is $5.2 \mathrm{~dB}$. A 54-fF series capacitor was placed at the HBT base to stabilize the array. The stabilized circular function is also plotted in Fig. 7. Simulations indicate that the grid is stable with a gain margin of $6.6 \mathrm{~dB}$ and a phase margin of $58^{\circ}$. Experimentally, this grid was stable for all bias conditions and achieved a peak gain of $5 \mathrm{~dB}$ at $40 \mathrm{GHz}$. The maximum output power was $0.67 \mathrm{~W}$.

\section{Resistive Stabilization}

A different approach was needed to stabilize a hybrid 100element pHEMT grid amplifier [4]. The pHEMT differential- 


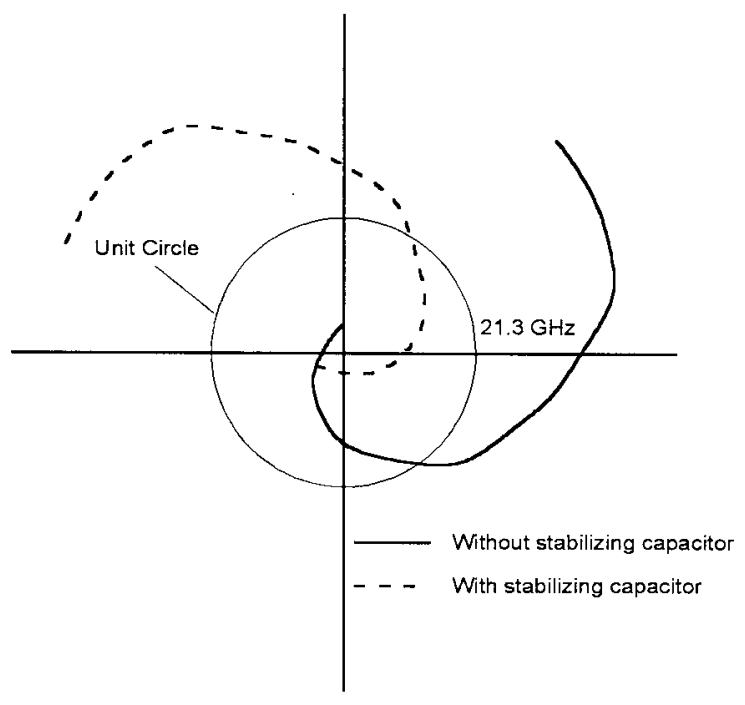

Fig. 7. Simulated circular function of a monolithic HBT grid amplifier with and without stabilizing capacitors.

TABLE I

Comparison of Simulated Gain Margins and Measured ONSET OSCILlATION CURRENTS FOR THE HBT AMPLIFIER Grid With and Without Stabilizing CAPacitors

\begin{tabular}{c|c|c}
\hline $\begin{array}{c}\text { Stabilizing Capacitor } \\
(\mathrm{pF})\end{array}$ & $\begin{array}{c}\text { Gain Margin } \\
(\mathrm{dB})\end{array}$ & $\begin{array}{c}\text { Onset Current } \\
(\mathrm{mA})\end{array}$ \\
\hline None & -5.4 & 2.4 \\
\hline 0.7 & -3.9 & 3 \\
\hline 0.36 & -1.5 & 4 \\
\hline 0.18 & 1.9 & 6 \\
\hline 0.1 & 3.4 & $>10$ \\
\hline
\end{tabular}

pair chips were fabricated by Lockheed Martin Laboratories, Baltimore, MD. This array also suffered from common-mode oscillations when the bias current was increased beyond $9 \mathrm{~mA}$ per transistor-well below the device's usual operating current. The amplifier gain at the onset of instability was only $3 \mathrm{~dB}$. The oscillation frequency varied between $8-9 \mathrm{GHz}$, depending on the bias. This oscillation was insensitive to elements external to the active array. The radiated power, shown in Fig. 8, was strongest in the plane of the grid and polarized along the output drain leads. Most of the power was concentrated toward the gate bias side. Again, we concluded that the oscillation was a common-mode checkerboard one, similar to the instability previously discussed.

The equivalent circuit for the oscillation is shown in Fig. 9(a). The pHEMT array did not have any gap capacitors in the input lead. Again, the length of the transmission lines is calculated using the 7.3-mm unit-cell size and an assumed effective dielectric constant between the substrate $\left(\varepsilon_{r}=2.2\right)$ and air. The transmission-line impedance is chosen empirically, as is the inductance of the source bias line. The substrate radiation resistance is not included in the simulation. The stability analysis predicts an oscillation at $8.9 \mathrm{GHz}$, as shown in Fig. 9(b). The zero-phase circular function is $1.2 \mathrm{~dB}$. This is in good agreement with the measured oscillation frequency.

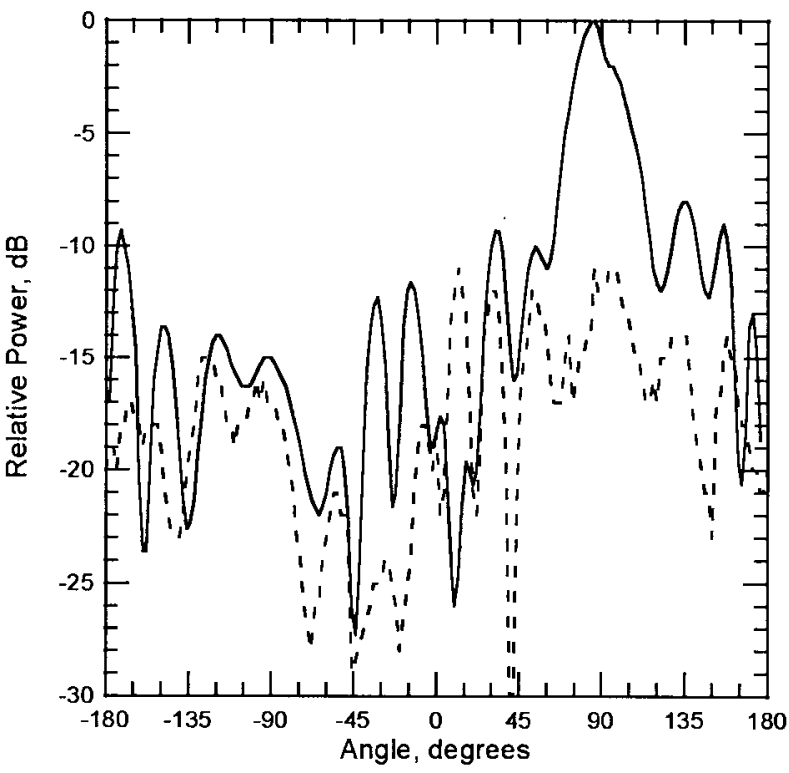

Fig. 8. Measured oscillation radiation pattern for the 100-element pHEMT grid. The pattern is taken in the amplifier's output $H$-plane. The solid line is polarized along the grid's output drain leads. The dashed line is polarized along the input gate leads.

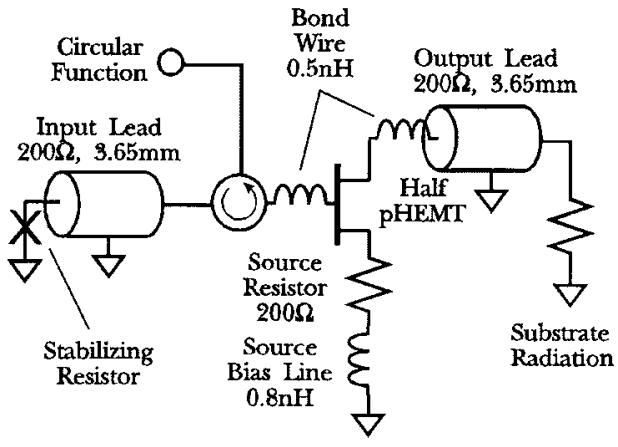

(a)

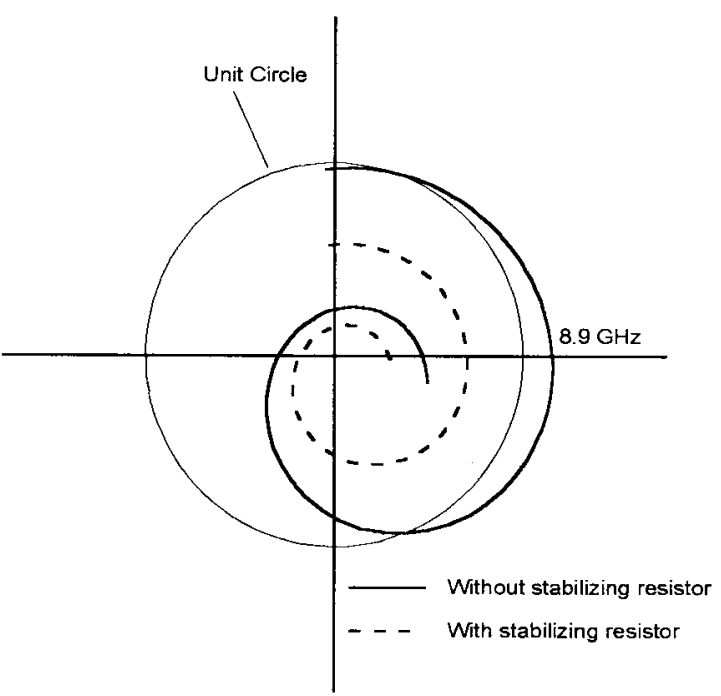

(b)

Fig. 9. (a) Transmission-line equivalent circuit for the 100-element pHEMT grid oscillation. (b) Simulated circular function of the grid with and without stabilizing resistors. 


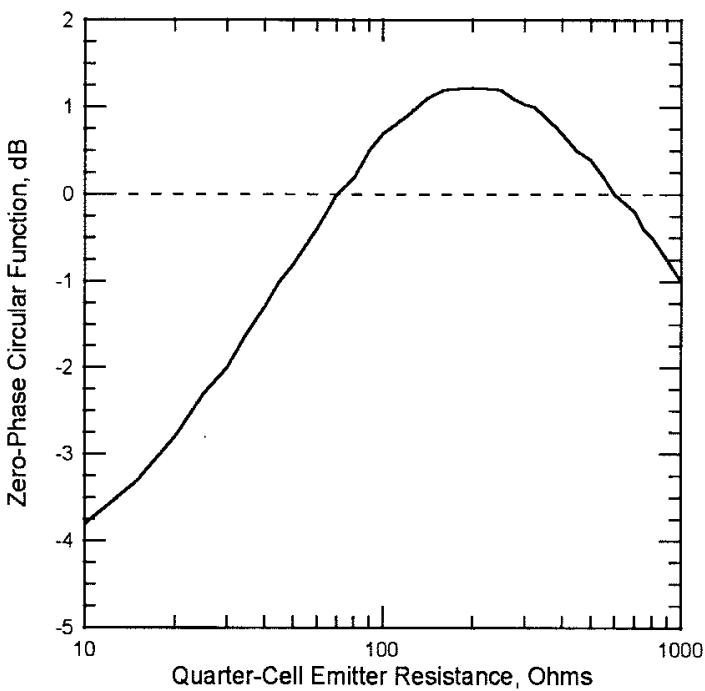

Fig. 10. Zero-phase circular function versus quarter-cell source resistance for the 100-element pHEMT grid of Fig. 9. The gate stabilization resistor is not included in the simulation.

Unlike the HBT arrays, the pHEMT grid could not be stabilized with a capacitor. This is because the pHEMT chips were not self-biased. An independent gate bias supplied along the grid's horizontal gate lead was necessary. Instead, this grid was stabilized by adding series $50-\Omega$ chip resistors in the gate leads at the node marked with an "X," midway between the active chips. This is possible because the pHEMT's draw very little gate current. These resistors reduce the magnitude of the circular function. Fig. 9(b) also plots the circular function for the stabilized grid. The gain margin is $3.0 \mathrm{~dB}$. With the resistors in place, the grid could be biased to a current of $15 \mathrm{~mA}$ per device, and the gain increased to $12 \mathrm{~dB}$ at $9 \mathrm{GHz}$. The saturated output power was $3.7 \mathrm{~W}$. Unfortunately, the stabilizing resistors will degrade the gain by $1.5 \mathrm{~dB}$ and the noise figure by $0.8 \mathrm{~dB}$. When possible, reactive stabilization is preferable.

\section{Reducing Common-Mode Resistance}

The original intent of including emitter or source resistance in the differential pair was to reduce the pair's common-mode gain and thus prevent common-mode oscillations. Ironically, our stability model suggests that this common-mode resistance is at least partly responsible for the amplifier's instability. Consider the 100-element pHEMT amplifier discussed previously. Fig. 10 plots the zero-phase circular function against the quarter-cell common-mode source resistance. The gate stabilizing resistors are not included. This result indicates that removing the source resistance would actually stabilize the grid. Removing these resistors would also increase the amplifier's efficiency. In the pHEMT grid, up to $40 \%$ of the total bias power is lost in the source resistors, limiting the peak power-added efficiency to $12 \%$ [4]. For the monolithic HBT amplifier, $66 \%$ of the bias power is lost in the emitter resistors, resulting in an efficiency of only $4 \%$ and large temperature rises [6]. Removal of the common-mode resistance would be a key to increasing the efficiency of grid amplifiers.

This idea was investigated on a 16-element hybrid high electron-mobility transistor (HEMT) array [5]. The

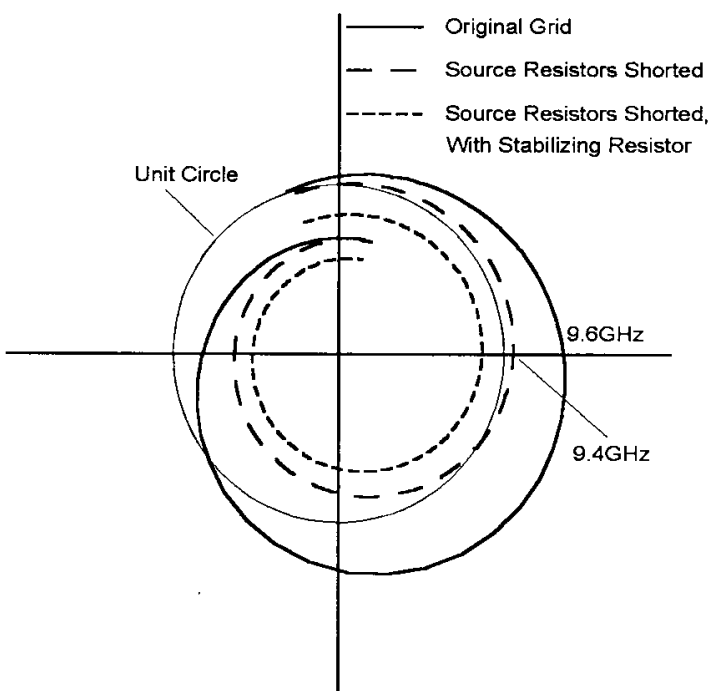

Fig. 11. Simulated circular function of a 16-element hybrid HEMT grid amplifier [5].

differential-pair chips were provided by the NASA Jet Propulsion Laboratory, Padadena, CA. Each transistor had a $50-\Omega$ common-mode source resistor. Like the other grids, this original array suffered from a common-mode oscillation at a relatively low-bias current. The oscillation frequency was between 9-10 GHz. The stability model, shown in Fig. 11, predicts an oscillation at $9.6 \mathrm{GHz}$, with a zero-phase circular function magnitude of $2.7 \mathrm{~dB}$.

To stabilize the grid, the $50-\Omega$ source resistors were shorted with bond wires. We suspect that the bond wires did not completely eliminate the source resistance, but reduced it to $2.5 \Omega$. The circular function for the grid with the source resistors shorted is also plotted in Fig. 11. Theory predicts that the grid is still unstable at $9.4 \mathrm{GHz}$, but the magnitude of the circular function is greatly reduced to $0.5 \mathrm{~dB}$. Experimentally, shorting the source resistors did not entirely suppress the spurious oscillation, but did reduce its power by $10 \mathrm{~dB}$. The grid could be completely stabilized by adding $20-\Omega$ resistors in the gate leads, midway between active chips. Fig. 11 also plots the stabilized circular function. The gain margin is $1.2 \mathrm{~dB}$.

The stabilized grid had a measured peak gain of $11 \mathrm{~dB}$ at $10 \mathrm{GHz}$. Reducing the common-mode source resistors had a considerable effect on the amplifier's efficiency, as shown in Fig. 12. This amplifier delivered $160 \mathrm{~mW}$ with a peak poweradded efficiency of $23 \%$. This is a factor of two more efficient than the 100-element pHEMT grid [4], and is the highest efficiency ever measured in a grid amplifier.

\section{CONCLUSION}

A stability model for grid amplifiers has been presented. This model successfully predicts common-mode instabilities often seen in amplifier grids. Three techniques for stabilizing the grid have been discussed. Capacitors can be placed in the input leads of self-biased HBT arrays to suppress the spurious oscillations. Resistive stabilization has been successful in pHEMT grids. Finally, the amplifiers may be stabilized by reducing or eliminating the common-mode resistance originally thought necessary to prevent common-mode oscillations. This 


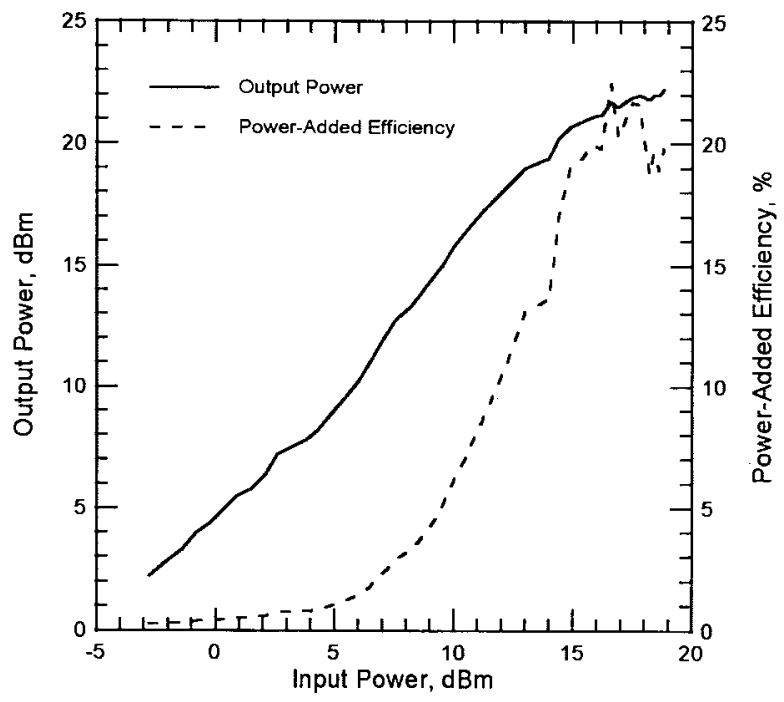

Fig. 12. Output power and power-added efficiency for the 16-element amplifier with source resistors removed. This is the most efficient grid amplifier ever reported.

final approach will greatly increase the amplifier's efficiency. Experimental evidence has been presented to validate the stability model. This model is currently being used as a tool to design stable hybrid and monolithic grid amplifiers. Rapid improvements in finite-element computer-aided design (CAD) software will allow us to further refine our stability model.

\section{ACKNOWLEDGMENT}

The authors would like to thank E. A. Sovero of Rockwell Science Center, Thousand Oaks, CA, S. W. Duncan of Lockheed Martin, Nashua, NH, and R. P. Smith of NASA Jet Propulsion Laboratory, Pasadena, CA. The authors are also grateful to J. J. Rosenberg of Harvey Mudd College, Claremont, CA, for many insightful discussions.

\section{REFERENCES}

[1] M. Kim, J. J. Rosenberg, R. P. Smith, R. M. Weikle, J. B. Hacker, M. P. De Lisio, and D. B. Rutledge, "A grid amplifier," IEEE Microwave Guided Wave Lett., vol. 1, pp. 322-324, Nov. 1991.

[2] M. Kim, E. A. Sovero, J. B. Hacker, M. P. De Lisio, J.-C. Chiao, S.-J. Li, D. R. Gagnon, J. J. Rosenberg, and D. B. Rutledge, "A 100-element HBT grid amplifier," IEEE Trans. Microwave Theory Tech., vol. 41, pp. 1762-1771, Oct. 1993.

[3] C.-M. Liu, E. A. Sovero, M. P. De Lisio, A. Moussessian, J. J. Rosenberg, and D. B. Rutledge, "Gain and stability models for HBT grid amplifiers," in IEEE AP-S Int. Symp. Dig., Newport Beach, CA, June 1995, pp. 1292-1295.

[4] M. P. De Lisio, S. W. Duncan, D.-W. Tu, C.-M. Liu, A. Moussessian, J. J. Rosenberg, and D. B. Rutledge, "Modeling and performance of a 100-element pHEMT grid amplifier," IEEE Trans. Microwave Theory Tech., vol. 44, pp. 2136-2144, Dec. 1996.

[5] A. Moussessian, "Quasioptical active antennas," Ph.D. dissertation, Dept. Elect. Eng., California Inst. Technol., Pasadena, CA, 1996.

[6] C.-M. Liu, E. A. Sovero, W.-J. Ho, J. A. Higgins, M. P. De Lisio, and D. B. Rutledge, "Monolithic 40-GHz 670-mW HBT grid amplifier," in IEEE MTT-S Int. Microwave Symp. Dig., San Francisco, CA, June 1996, pp. 1123-1126.

[7] M. P. De Lisio, S. W. Duncan, D.-W. Tu, S. Weinreb, C.-M. Liu, and D. B. Rutledge, "A 44-60 GHz monolithic pHEMT grid amplifier," in IEEE MTT-S Int. Microwave Symp. Dig., San Francisco, CA, June 1996, pp. $1127-1130$.

[8] D. W. Griffin, "Monolithic active array limitation due to substrate modes," in IEEE AP-S Int. Symp. Dig., Newport Beach, CA, June 1995 pp. $1300-1303$

[9] P. Preventza, M. Matloubian, and D. B. Rutledge, "A 43-GHz AlInAs/GaInAs/InP HEMT grid oscillator," in IEEE MTT-S Int. Microwave Symp. Dig., Denver, CO, June 1997, pp. 1057-1060.

[10] R. D. Martinez and R. C. Compton, "A general approach for the $S$-parameter design of oscillators with 1- and 2-port active devices," IEEE Trans. Microwave Theory Tech., vol. 40, pp. 569-574, Mar. 1992.

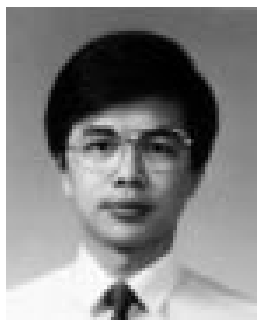

Cheh-Ming (Jeff) Liu received the B.S. and M.S degrees in electrical engineering from National Chiao-Tung University, Hsin-Chu, Taiwan, R.O.C. in 1986 and 1988, respectively, and the M.S. and $\mathrm{Ph} . \mathrm{D}$. degrees in electrical engineering from the California Institute of Technology, Pasadena, in 1994 and 1996, respectively.

Since 1996, he has been a Member of the technical staff in the Mixed-Signal High-Speed Circuits Department, Rockwell Science Center, Rockwell International Corporation, Thousand Oaks, CA. His research interests include antenna module design, RF and highspeed package modeling, and high-resolution sigma-delta analog-to-digital converter (ADC) development.

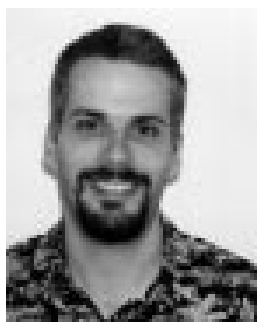

Michael P. De Lisio (S'90-A'95-M'96) was born in Southfield, MI, on July 29, 1968. He received the B.S.E. degree in electrical engineering from the University of Michigan at Ann Arbor, in 1990, the M.S. degree in electrical engineering from the California Institute of Technology, Pasadena, in 1991, and the Ph.D. degree from Caltech, in 1996.

In 1996, he joined the Department of Electrical Engineering, University of Hawaii at Manoa, as an Assistant Professor. His research interests include high-frequency solid-state devices, microwave and millimeter-wave power combining, and monolithic quasi-optical devices.

Dr. De Lisio is a member of Tau Beta Pi and Eta Kappa Nu.

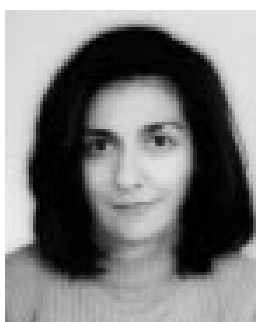

Alina Moussessian was born in Tehran, Iran, on June 21,1966 . She received the B.S. degree in electrical engineering from the Iran University of Science and Technology, Tehran, Iran, in 1988, and the M.S. and Ph.D. degrees in electrical engineering from the California Institute of Technology, Pasadena, in 1992, and 1997, respectively.

In 1997, she joined the Radar Science and Engineering Section, Jet Propulsion Laboratory, Pasadena, CA. Her research interests include microwave, millimeter-wave, and submillimeterwave power combining, beam-steering, and $\mathrm{CAD}$ and measurement of microwave circuits.

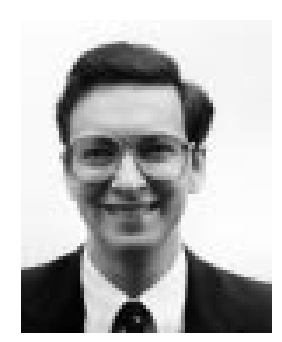

David B. Rutledge (S'77-M'77-SM'89-F'93) is a Professor of electrical engineering at the California Institute of Technology, Pasadena. His research has been in developing integrated-circuit antennas, imaging arrays, active grids, and software for $\mathrm{CAD}$ and measurement. His group has contributed 200 publications to the technical literature. He has co-authored the widely distributed educational microwave CAD package Puff with over 12000 copies worldwide. Five of his students have won Presidential Investigator and Career Awards.

Prof. Rutledge was a Distinguished Lecturer for the IEEE Antennas \& Propagation Society. He was the recipient of the IEEE Microwave Theory \& Techniques Society Microwave Prize and the Teaching Award of the Associated Students of Caltech. 